\title{
REDUÇÃO DE STENOPHALIUM A. ANDERB. À SINONÍMIA DE ACHYROCLINE (LESS.) DC. (ASTERACEAE-GNAPHALIEAE) ${ }^{1}$
}

\author{
LEONARDO PAZ DEBLE ${ }^{2}$ JOSÉ NEWTON CARDOSO MARCHIORI $^{3}$
}

\section{RESUMO}

A análise comparativa das estruturas morfológicas (hábito, folhas, tipo de indumento, disposição da inflorescência, receptáculo, invólucro, brácteas involucrais, ausência ou número de flores femininas, e presença de pêlos nos aquênios) utilizadas para diferenciar Achyrocline (Less.) DC. de Stenophalium A. Anderb. demonstram a existência de variação contínua, impossibilitando uma clara separação entre ambos os gêneros. Stenophalium foi presentemente reduzido à sinonímia de Achyrocline, sendo propostas quatro novas combinações: Achyrocline chionaea (DC.) Deble \& Marchiori, Achyrocline eriodes (Mattf.) Deble \& Marchiori, Achyrocline gardneri (Baker) Deble \& Marchiori e Achyrocline heringeri (H. Rob.) Deble \& Marchiori. São também fornecidas ilustrações de flores femininas, pêlos, estigma e pápus.

Palavras-chave: Achyrocline, Stenophalium, Asteraceae, Gnaphalieae.

\section{ABSTRACT}

A comparison of morphological features (habit, leaf, indumentum, arrangement of synflorescence, receptacle, involucre, involucral bracts, missing or number of female flowers, glandular hairs on the achenes) that are commonly used to set apart Achyrocline (Less.) DC. from Stenophalium A. Anderb. shows a continuous variation, making impossible a clear separation between these genera. Stenophalium is presently reduced to the synonymity of Achyrocline and four new combinations are proposed: Achyrocline chionaea (DC.) Deble \& Marchiori, Achyrocline eriodes (Mattf.) Deble \& Marchiori, Achyrocline gardneri (Baker) Deble \& Marchiori and Achyrocline heringeri (H. Rob.) Deble \& Marchiori. Illustrations of female flowers, hairs, style and pappus are also furnished.

Key words: Achyrocline, Stenophalium, Asteraceae, Gnaphalieae.

\section{INTRODUÇÃO}

O gênero Stenophalium foi concebido por Anderberg (1991) para abrigar três espécies brasileiras, subordinadas, de maneira insatisfatória, ao gênero africano Stenocline: S. chionaea, $S$. eriodes e $S$. heringeri. A revisão dos gêneros brasileiros da tribo Gnaphalieae demonstrou que Stenophalium, em verdade, está estreitamente relacionado a Achyrocline. A análise sobre seu real posicionamento dentro da tribo Gnaphalieae, é o objeto do presente artigo.

$\mathrm{Na}$ descrição do gênero Stenocline, De Candolle (1838) dividiu o mesmo em três seções: Trichephalum, Eustenocline e ? Chionolepis, as duas primeiras abrigando espécies africanas e, a última, uma espécie brasileira (Stenocline chionaea).

Em trabalhos posteriores, três novas espécies foram acrescentadas a Stenocline: S. gardneri (Baker, 1882), S. eriodes (Mattfeld, 1927) e $S$. heringeri (Robinson, 1984).

Dillon \& Sagástegui (1991) apresentaram uma sinopse dos gêneros sul-americanos de Gnaphalinae. Os autores reconheceram como prematura a segregação das espécies brasileiras de Stenocline em um gênero distinto (Stenophalium), tendo em vista que os limites genéricos entre Achyrocline, Pseudognaphalium

1 Artigo recebido em 25/04/2005 e aceito para publicação em 26/06/2005.

2 Biólogo, Msc., bolsista da CAPES, doutorando do Curso de Pós-graduação em Engenharia Florestal, Centro de Ciências Rurais, Universidade Federal de Santa Maria, CEP 97105-900, Santa Maria (RS).deble.biol@bol.com.br

3 Engenheiro Florestal, Dr., bolsista de Produtividade em Pesquisa do CNPq, Professor Titular do Departamento de Ciências Florestais, Universidade Federal de Santa Maria, CEP 97105-900, Santa Maria (RS). balduinia@mail.ufsm.br 
e Stenocline ? Chionolepis não estão bem definidos. Os autores sugerem que os três gêneros anteriormente citados poderiam ser congenéricos e, nesse caso, Achyrocline deveria prevalecer como nome válido.

Hind (1993) descreveu uma nova espécie Stenophalium almasense-, e tranferiu Stenocline gardneri para o gênero de Anderberg. No mesmo trabalho, o autor reconheceu que Stenophalium é estreitamente relacionado a Achyrocline (Less.) DC. e a Pseudognaphalium Kirp., diferindo, todavia, por abrigar subarbustos com maior número de flores hermafroditas. Para o mesmo autor, tanto Pseudognaphalium como Achyrocline incluem ervas anuais e perenes, com maior número de flores femininas.

\section{COMPARAÇÃO ENTRE OS GÊNEROS}

\section{Hábito}

Para Hind (1993), Stenophalium compreende subarbustos, enquanto Achyrocline e Pseudognaphalium incluem ervas anuais e perenes. Em verdade, a presença de subarbustos é igualmente reportada para espécies de Achyrocline, como A. disjuncta, A. marchiorii, A. satureioides e A. luisiana.

Ambos os gêneros abrigam tanto ervas perenes (Achyrocline anabelae, A. hyperchlora, $A$. tomentosa, Stenophalium heringeri), como subarbustos (Achyrocline disjuncta, A. marchiorii, Stenophalium eriodes, S. gardneri), sejam eles pouco ramosos (Achyrocline gertiana, Stenophalium heringeri) ou densamente ramosos (Achyrocline brittoniana, A. satureioides, Stenophalium eriodes, S. gardneri), e com ramos eretos (Achyrocline alata, $A$ flaccida, Stenophalium heringeri), ascendentes (A luisiana, Stenophalium eriodes, $S$. chionaeum), prostados (Achyrocline marchiorii, $A$. rupestris e indivíduos pequenos de Achyrocline tomentosa e Stenophalium eriodes) $\mathrm{e}$, até mesmo, escandentes (Achyrocline scandens).

\section{Folhas}

Quanto à forma, as folhas podem ser lineares (Achyrocline satureioides, Stenophalium gardneri), lanceoladas (Achyrocline flaccida, Stenophalium heringeri), elípticas (Achyrocline hyperchlora, Stenophalium eriodes) e até ovadas (Achyrocline celosioides, Stenophalium chionaeum). Sempre alternas, os entre-nós são muito aproximados nos ramos (Achyrocline brittoniana, Achyrocline satureioides, Stenophalium gardneri), ou então relativamente afastados (Achyrocline gertiana, Stenophalium chionaeum). Podem ser concolores (Achyrocline satureioides, Stenophalium eriodes) ou discolores (Achyrocline ribasiana, Stenophalium chionaeum), de margens íntegras ou suavemente erosas, e 1-5-nervadas, com poucas nervuras secundárias; estas, partindo próximo à base, podem ser bem visíveis em algumas espécies de Achyrocline (A. venosa, A. glandulosa), apesar de, na maioria das vezes, resultarem ocultas pelo tomento (Achyrocline marchiorii, A. tomentosa, Stenophalium eriodes). Sésseis, de ápice agudo ou obtuso e base normalmente atenuada ou longo-atenuada em pseudopecíolo, podem ser longamente decurrentes, estendendo-se ao longo do caule, como em algumas espécies de Achyrocline (A. alata, A. arrojadoana e $A$. glandulosa) ou, então, apenas curtodecurrentes (Achyrocline madioides, Stenophalium eriodes); somente em Stenophalium chionaeum, S. gardneri e nas folhas superiores de Achyrocline brittoniana, observam-se folhas suavemente amplexicaules.

\section{Indumento}

O indumento possui valor taxonômico, principalmente quando relacionado a outros caracteres. Em geral, a presença de um tipo de pêlo exclui outro, dentro de uma mesma espécie. Em nível genérico, a presença ou ausência de determinado tipo de pêlo pode 
ser usada como auxílio para a separação de gêneros (Anderberg, 1991; Hilliard \& Burtt, 1981, Zardini, 1985).

Em folhas e ramos: Em Achyrocline e Stenophalium foram observados dois tipos de pêlos: glandulares e bisseriados, e não-glandulares e unisseriados com célula terminal longa, em forma de látigo ou flagelo (Figura 1); esta, medindo normalmente vários milímetros de comprimento, confere aspecto lanoso à pubescência. Em algumas espécies de Achyrocline, tais como em A. gertiana, A. glandulosa e A. hyperchlora, a célula terminal é caduca na parte adaxial da folha, acarretando aspecto laxamente lanoso e textura áspera. A pubescência pode ser incano-tomentosa (Achyrocline marchiorii, Stenophalium eriodes) ou, mais comumente, ferrugínea até gríseotomentosa, por vezes com pêlos alvos e gríseos, entremesclados (Achyrocline ribasiana). Adensidade do tomento é bastante variada, inclusive dentro de uma mesma espécie.

Nas brácteas involucrais: São reportados dois tipos de pêlos: glandulosos e bisseriados, e não-glandulosos e unisseriados. As brácteas externas e medianas normalmente apresentam ambos os tipos entremesclados, havendo, nas internas, apenas pêlos glandulosos. Entre distintas espécies, observou-se somente pequena variação no número e tamanho das células que compõem os pêlos.

Nas flores: Ambos os gêneros compartilham o mesmo tipo de pêlos - bisseriados e glandulares-, restritos aos lóbulos da corola, ocorrendo variações somente no que se refere ao número e tamanho das células (Figura 2). $\mathrm{O}$ tubo da corola apresenta-se glabro.

Nos aquênios: Foi reportada a presença de pêlos do tipo "twin-hairs", no epicarpo de algumas espécies. Tais pêlos, de forma globosa e desprovidos de células basais, podem ser obtusos (Achyrocline marchiori) ou agudos (Stenophalium gardneri).

\section{Inflorescência}

Os capítulos, reunidos em glomérulos, compõem cimas que formam corimbos terminais. Estes podem ser contraídos (Achyrocline marchiorii, A. satureioides, Stenophalium heringeri) ou não (Achyrocline luisiana, Stenophalium gardneri), dispondo-se, por vezes, ao longo dos ramos terminais, compondo amplas panículas (Achyrocline anabelae, A. gertiana, Stenophalium chionaeum).

\section{Invólucro}

Estreitamente cilíndrico (Achyrocline satureioides, Achyrocline flaccida), cilíndrico (Achyrocline luisiana, Achyrocline marchiorii, Achyrocline satureioides, Stenophalium gardneri) ou oblongo (Achyrocline gertiana, A. crassiuscula, Stenophalium chionaeum, $S$. eriodes, $S$. heringerii), o invólucro apresenta coloração muito variada, inclusive dentro de uma mesma espécie, podendo ser marfim (Achyrocline glandulosa, A. tomentosa, Stenophalium chionaeum, S. eriodes), creme (Achyrocline luisiana, A. tomentosa, Stenophalium gardneri, S. heringeri), amarelo (Achyrocline alata, A anabelae, A. flaccida, A. marchiorii, Stenophalium gardneri), dourado (Achyrocline satureioides) ou ferrugíneo (Achyrocline rufescens, A. satureioides), independentemente do estado de maturação.

\section{Receptáculo}

Plano e alveolado, o receptáculo é desprovido de páleas e fimbrias, assemelhando-se em todas as espécies.

\section{Brácteas involucrais}

Estreitamente imbricadas ou apenas imbricadas, as brácteas involucrais são escariosas e dispostas em (2) 3-5-séries. O número de brácteas no invólucro varia de 8 a 18 , não tendo relação com o número de flores (Tabela 1). Diferentes tipos de pêlos (ver Indumento) podem ser observa- 


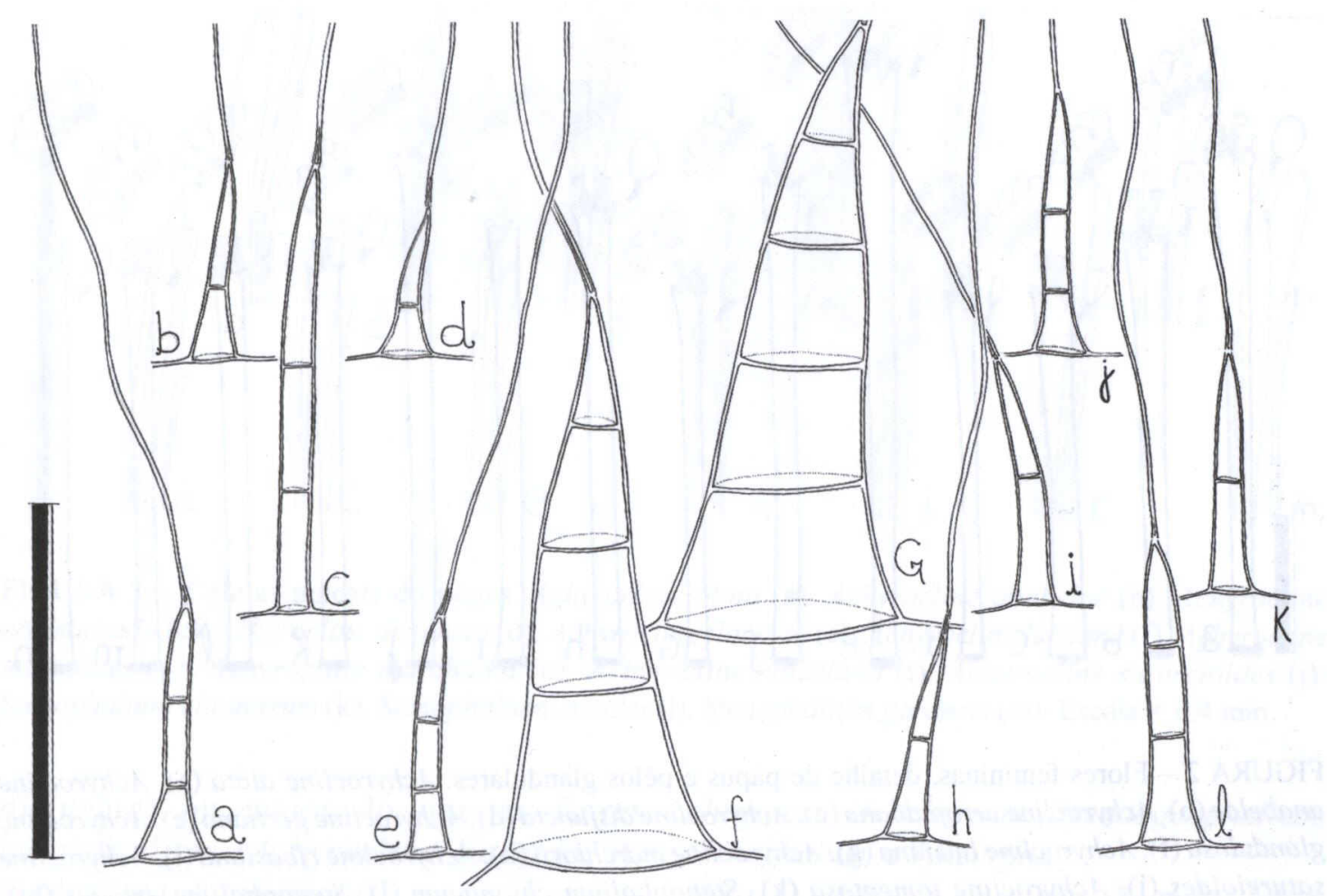

FIGURA 1 - Pêlos unisseriados da parte adaxial da folha. Achyrocline alata (a). Achyrocline anabelae (b). Achyrocline arrojadoana (c). Achyrocline disjuncta (d). Achyrocline crassiuscula (e). Achyrocline gertiana (f). Achyrocline glandulosa (g). Achyrocline marchiorii (h). Achyrocline satureioides (i). Stenophalium chionaeum (j). Stenophalium eriodes (k). Stenophalium gardneri (1). Escala $=0,2 \mathrm{~mm}$.

dos. Com relação à forma, as brácteas involucrais externas podem ser ovadas, ovadolanceoladas ou elípticas, sendo geralmente mais curtas do que as medianas e internas (Achyrocline anabelae, A. satureioides, Stenophalium gardneri); em alguns casos são de igual tamanho (Achyrocline glandulosa, A. tomentosa, Stenophalium chionaeum, $S$. eriodes), mas raramente mais longas ( $A$. disjuncta). As medianas e internas podem ser ovado-lanceoladas (Achyrocline tomentosa, Stenophalium gardneri), lanceoladas (Achyrocline arrojadoana, Stenophalium chionaeum), oblongas (Achyrocline anabelae, Stenophalium eriodes) ou, mais raramente, oblongo-espatuladas (Achyrocline luisiana,
Stenophalium gardneri), oblongo-lineares (Achyrocline ribasiana) e linear-lanceoladas (Achyrocline gertiana).

Estereoma: Como porção basal e cartilaginosa das brácteas involucrais, o estereoma pode ser dividido ou não, fornecendo importante caráter para a distinção de gêneros (Hilliard \& Burtt, 1981; Anderberg, 1991). No complexo Achyrocline-Pseudognaphalium-Stenophalium, o estereoma é dividido e de estrutura bastante semelhante, o que levou Anderberg (1991) a sugerir uma possível relação entre os mesmos.

\section{Flores}

Na concepção original do gênero Stenocline, De Candole (1838) refere: "capitula 1-5-flora 


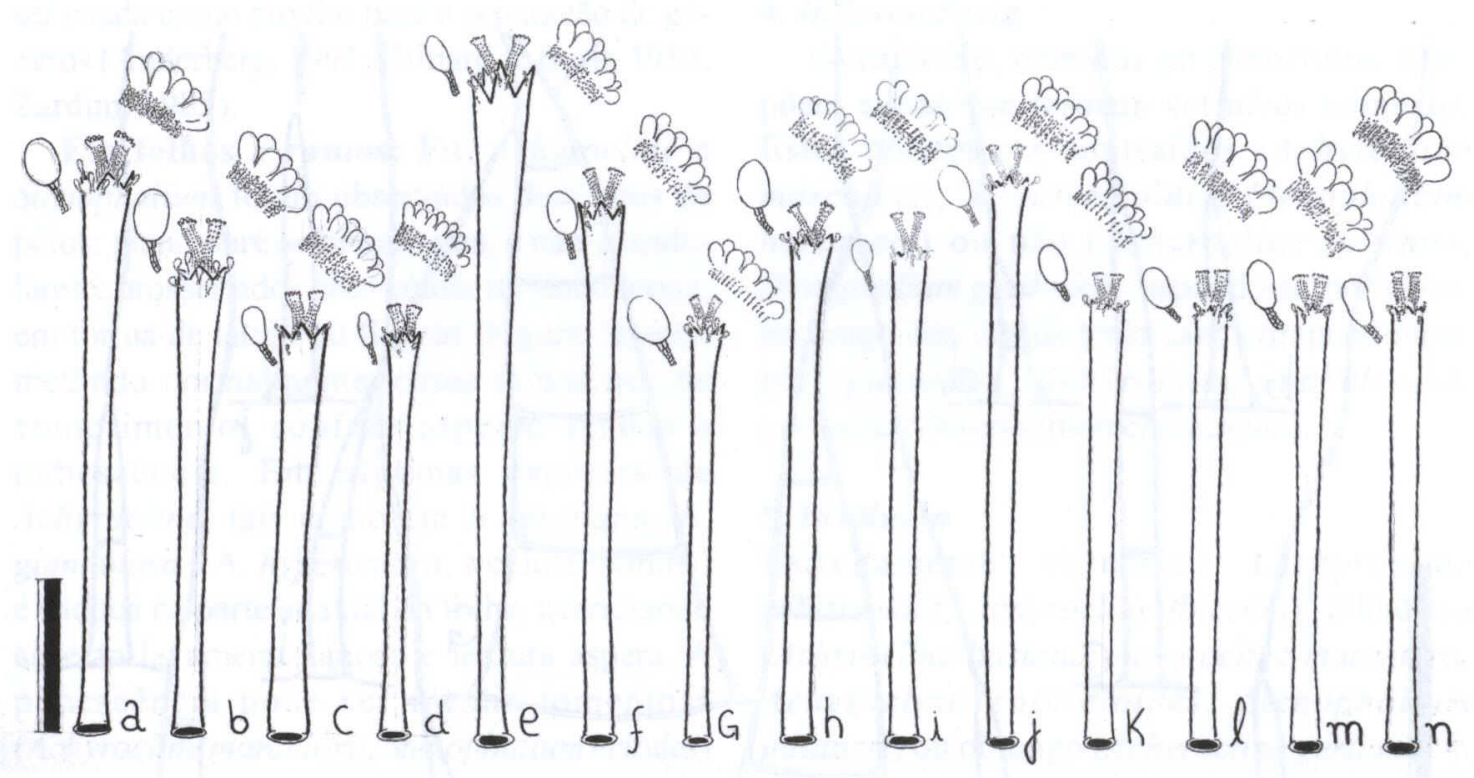

FIGURA 2 - Flores femininas, detalhe de pápus e pêlos glandulares. Achyrocline alata (a). Achyrocline anabelae (b). Achyrocline arrojadoana (c). Achyrocline disjuncta (d). Achyrocline gertiana (e). Achyrocline glandulosa (f). Achyrocline luisiana (g). Achyrocline marchiorii (h). Achyrocline ribasiana (i). Achyrocline satureioides (j). Achyrocline tomentosa (k). Stenophalium chionaeum (1). Stenophalium eriodes (m). Stenophalium gardneri $(\mathrm{n})$. Escala $=1 \mathrm{~mm}$.

homogama oblonga...", considerando-o "genus forte dividendum...". Para a seção ? Chionolepis, por sua vez, ele citou "capitula 4-5 flora homogama...".

Na diagnose de Stenophalium, Anderberg (1991) descreveu: "capitula homogama, discoidea... flosculi feminei destituti...". Do exposto, verifica-se que De Candolle e Anderberg, ao conceberem Stenocline ? Chionolepis e Stenophalium, reconheceram capítulos homógamos, destituídos de flores femininas. $\mathrm{O}$ estudo mais amplo do complexo AchyroclineStenophalium, todavia, demonstra que as espécies apresentam variação no número de flores hermafroditas e femininas, não justificando a separação de ambos os gêneros com base nesse caráter (Tabela 1).

Características gerais: Corola perpendicular ao aquênio, de coloração creme ou amarela, com lóbulos eretos, providos de pêlos glandulares e venação até próximo ao ápice (Figura 2).

Flores femininas: Marginais, dispostas em uma única série e em número reduzido (0-12). A corola, tubuloso-filiforme ou filiforme, de 2$5,5 \mathrm{~mm}$ de comprimento, pode ter base dilatada ou não (esta característica, em geral está relacionada com o grau de maturidade dos aquênios); o ápice, por sua vez, é glanduloso e 4-dentado. Os dentes, deltóides e medindo 0,03-0,2 mm, têm ápice normalmente agudo, mais raramente acuminado (Achyrocline gertiana) ou obtuso (Achyrocline luisiana, Stenophalium gardneri). As flores femininas podem ser ausentes em alguns capítulos (Stenophalium gardneri, S. heringeri), ou em número menor (Achyrocline luisiana, A. guerreroana, Stenophalium gardneri, S. chionaeum, S. eriodes), igual (Achyrocline anabelae, A. guerreroana, A. 


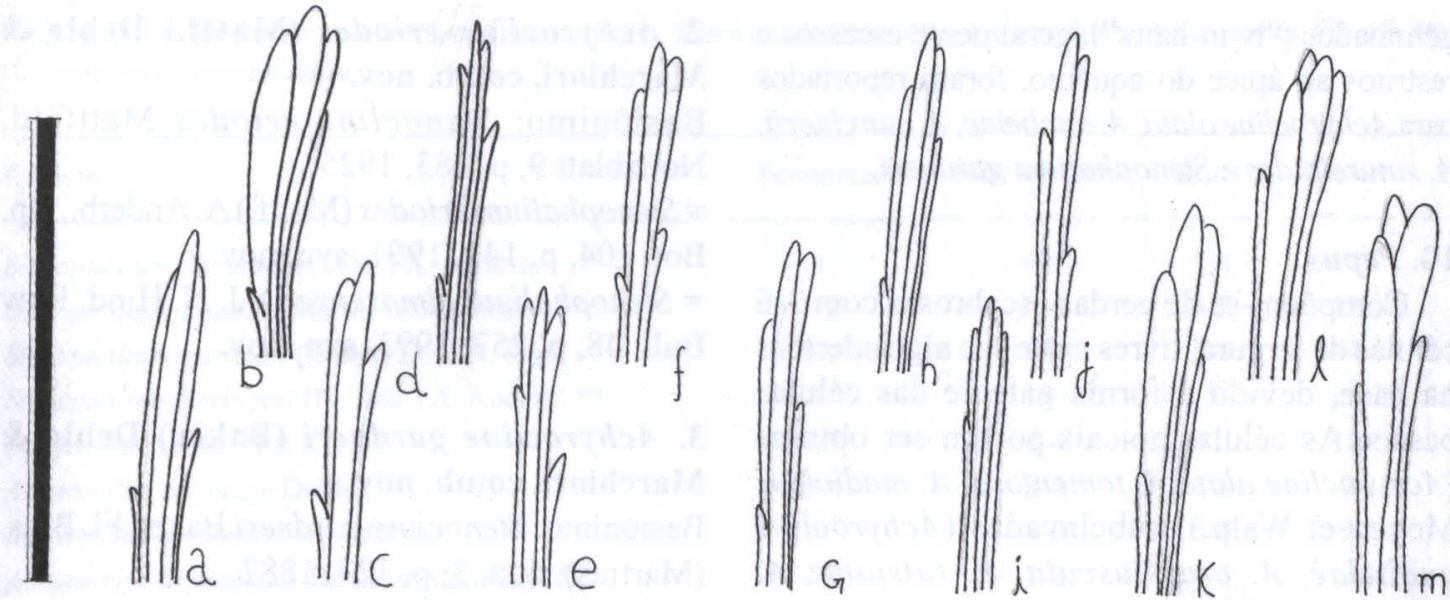

FIGURA 3 - Células apicais do pápus. Achyrocline alata (a). Achyrocline anabelae (b). Achyrocline crassiuscula (c). Achyrocline disjuncta (d). Achyrocline flaccida (e). Achyrocline luisiana (f). Achyrocline madioides (g). Achyrocline marchiorii (h). Achyrocline ribasiana (i). Achyrocline satureioides (j). Stenophalium chionaeum (k). Stenophalium eriodes (l). Stenophalium gardneri $(\mathrm{m})$. Escala = 0,4 mm.

disjuncta) ou maior do que as flores hermafroditas (Achyrocline flaccida, A. hyperchlora, A. tomentosa).

Flores hermafroditas: Poucas (0-6) e centrais. A corola, tubulosa e com 2-5 mm de comprimento, pode ter base dilatada ou não, e ápice glanduloso, 5-dentado ou 5-lobado. Dentes ou lóbulos deltóides, de 0,15-0,7 mm, com ápice normalmente agudo, mais raramente acuminado (Achyrocline gertiana). Flores hermafroditas podem não ocorrer em alguns capítulos de determinadas espécies (Achyrocline oaxacana $A$. venosa).

Estigma: Varia de 2,5 a $5 \mathrm{~mm}$ de comprimento, com ramas de $0,5-1 \mathrm{~mm}$, truncadas no ápice, de onde se eleva uma coroa de pêlos clavados, obtusos ou truncados (Figura 2).

Anteras: Variam de 1,2 a $2 \mathrm{~mm}$; são caudadas, laciniadas, com conectivo prolongando-se em apêndice triangular, hialino, levemente agudo ou obtuso. A forma das anteras possui valor taxonômico, pois dos 41 gêneros reconhecidos por Anderberg (1991) na subtribo Gnaphaliinae, apenas sete apresentam anteras caudadas: Achyrocline, Edmondia, Heli- chrysopis, Helichrysum, Pseudognaphalium, Stenophalium e Syncarpha.

Nota: O estigma e as anteras são muito semelhantes em todas as espécies estudadas.

Morfologia do pólen: É muito semelhante e "Gnaphalióide", em todos os gêneros da subtribo. Giangualani (1976) investigou o pólen das espécies argentinas de Achyrocline. Leins $(1971,1973)$ estudou a estrutura palinológica no complexo Inuleae sensu lato.

\section{Aquênios}

Pequenos (0,5-1 mm) e bivenosos, os aquênios podem ser elípticos (Achyrocline alata, A. anabelae, A. flaccida, Stenophalium gardneri), ovados (Achyrocline marchiorii, A. satureioides, Stenophalium chionaeum), oblongos (Achyrocline arrojadoana, A. gertiana, A. glandulosa), levemente comprimidos, ou, por vezes, angulosos (Achyrocline gertiana, Stenophalium gardneri), tendo superfície lisa (Achyrocline brittoniana, Stenophalium eriodes), finamente estriada (Achyrocline flaccida, Stenophalium chionaeum) ou papilosa (Achyrocline satureioides, A. marchiorii). Pêlos 
geminados ("twin-hairs"), geralmente escassos e restritos ao ápice do aquênio, foram reportados para Achyrocline alata, A. anabelae, A. marchiorii, A. satureioides e Stenophalium gardneri.

\section{Pápus}

Compõem-se de cerdas escabrosas com 3-6 células de largura, livres entre si e algo aderidas na base, devido à forma patente das células basais. As células apicais podem ser obtusas (Achyrocline alata A. tomentosa, A. madioides Meyen et Walp.), subclavadas (Achyrocline anabelae, A. crassiuscula, A. luisiana, A. ribasiana, Stenophalium chionaeum, S. eriodes) ou clavadas (Stenophalium gardneri) (Figura 3). Nota: Quanto à estrutura do pápus, Achyrocline disjuncta separa-se das demais espécies por apresentar células apicais agudas e células basais ascendentes.

\section{RESULTADOS}

Do acima exposto, fica evidente que Stenophalium é congenérico de Achyrocline. A comparação das estruturas morfológicas utilizadas para diferenciar ambos os gêneros (hábito, folhas, tipo de indumento, disposição da inflorescência, receptáculo, invólucro, brácteas involucrais, ausência ou reduzido número de flores femininas) demonstram uma variação contínua, impossibilitando a nítida separação entre os mesmos. Com base no princípio de prioridade, Achyrocline é gênero mais antigo (De Candolle, 1838), devendo Stenophalium (Anderberg, 1991), portanto, ser reduzido à sua sinonímia. Em conseqüência, tornam-se necessárias as seguintes novas combinações e sinonímias:

\section{Achyrocline chionaea (DC.) Deble \&} Marchiori, comb. nov.

Basiônimo: Stenocline chionaea DC., Prodromus 6, p. 219, 1838.

= Achyrocline rugosa Gardner, London J. Bot.

7, p. 419, 1848;

= Stenophalium chionaeum (DC.) A. Anderb., Op. Bot. 104, p. 141, 1991. syn. nov.
2. Achyrocline eriodes (Mattf.) Deble \& Marchiori, comb. nov.

Basiônimo: Stenocline eriodes Mattfeld, Notizblatt 9, p. 383, 1925.

= Stenophalium eriodes (Mattf.) A. Anderb., Op. Bot. 104, p. 141, 1991. syn. nov.

= Stenophalium almasense D. J. N. Hind, Kew Bull. 48, p. 257, 1993. syn. nov.

\section{Achyrocline gardneri (Baker) Deble \&} Marchiori, comb. nov.

Basiônimo: Stenocline gardneri Baker, Fl. Bras. (Martius), 6, n. 3, p. 127, 1882.

$=$ Achyrocline satureioides Gardner, London $\mathrm{J}$.

Bot. 7, p. 424, 1848 [non DC.];

= Stenophalium gardneri (Baker) D. J. N. Hind, Kew Bull. 48, p. 256, 1993. syn. nov.

\section{Achyrocline heringeri (H. Rob.) Deble \&} Marchiori, comb. nov.

Basiônimo: Stenocline heringeri H. Robinson, Phytologia 55, n. 3, p. 122, 1984.

$=$ Stenophalium heringeri (H. Rob.) A. Anderb., Op. Bot. 104, p. 141, 1991. syn. nov.

\section{BIBLIOGRAFIA}

Anderberg, A. Taxonomy and phylogeny of the tribe Gnaphalieae (Asteraceae). Opera Bot., Copenhagen, n. 104, p. 1-195, 1991.

Baker, J. F. Compositae. In: Martius, C. F., Flora Brasiliensis, München, Wien and Leipzig, v. 6, n. 3, p.1-132, 1882.

Deble, L. P. Novas espécies de Achyrocline (Less.) DC. para o sul do Brasil. Balduinia, Santa Maria, n. 3, p. 5-9, 2005.

Deble, L. P. Uma nova espécie de Achyrocline (Less.) DC. (Asteraceae) para o Rio Grande do Sul (Brasil). Balduinia, Santa Maria, n. 4, p. 1-4, 2005.

Deble, L. P., Marchiori, J. N. C. Novidades em Achyrocline (Less.) DC. (Asteraceae Gnaphalieae). Balduinia, Santa Maria, n. 3, p. 10-13, 2005.

Deble, L. P., Marchiori, J. N. C. Novas espécies de Achyrocline (Less.) DC. (Asteraceae) para o Brasil. Balduinia, Santa Maria, n. 4, p. 5-9, 2005.

De Candolle, A. P., Prodr. Syst. Nat. Reg. Veget., Paris, 6, p. 218-223, 1838. 
TABELA 1: Número de brácteas involucrais, flores femininas e hermafroditas em diferentes espécies dos gêneros Achyrocline e Stenophalium.

\begin{tabular}{|c|c|c|c|c|}
\hline Espécie & Femininas & Hermafroditas & Total & Brácteas \\
\hline Stenophalium chionaeum (DC.) A. Anderb. & 1 & $4-5$ & $5-6$ & 12 \\
\hline Stenophalium eriodes (Mattf.) A. Anderb. & $1-2$ & $5-6$ & $6-7$ & $12-14$ \\
\hline Stenophalium gardneri (Baker) D. J. N. Hind & $0-2$ & $2-4$ & $4-6$ & $14-16$ \\
\hline Stenophalium heringeri (H. Rob.) A. Anderb. ** & 0 & 5 & 5 & ca. 15 \\
\hline Achyrocline alata (H.B.K.) DC. & $4-7$ & $1-4$ & $7-10$ & $10-14$ \\
\hline Achyrocline anabelae Deble & $3-4$ & $2-4$ & $5-8$ & $9-11$ \\
\hline Achyrocline arrojadoana Mattf. & 4 & $1-2$ & $5-6$ & $9-10$ \\
\hline Achyrocline brittoniana Deble \& Marchiori * & $2-5$ & $1-2$ & $4-6$ & $9-10$ \\
\hline Achyrocline crassiuscula (Malme) Deble \& Marchiori & $8-10$ & $2-3$ & $11-13$ & $14-16$ \\
\hline Achyrocline disjuncta Hemsl. & 2 & 2 & 4 & $11-13$ \\
\hline Achyrocline flaccida (Weinm.) DC. & $4-5$ & $1-2$ & $5-7$ & $9-10$ \\
\hline Achyrocline gaudens V. Badillo \& Sanchéz * & $3-5$ & $1-2$ & $5-7$ & 8 \\
\hline Achyrocline gertiana Deble \& Marchiori & $7-10$ & $2-3$ & $10-12$ & $16-18$ \\
\hline Achyrocline glandulosa Blake & $4-5$ & 2 & $6-7$ & $8-9$ \\
\hline Achyrocline guerreroana Nesom *** & $1-3$ & $2-3$ & $4-5$ & $9-11$ \\
\hline Achyrocline hyperchlora Blake * & $3-4$ & 1 & $4-5$ & $9-11$ \\
\hline Achyrocline latifolia Weddel * & 3 & $1-2$ & $4-5$ & $10-12$ \\
\hline Achyrocline luisiana Deble & $1-2$ & 4 & $5-6$ & $8-9$ \\
\hline Achyrocline marchiorii Deble & $3-4$ & $1-3$ & $5-7$ & $12-13$ \\
\hline Achyrocline oaxacana Nesom *** & 4 & $0-1$ & $4-5$ & 9 \\
\hline Achyrocline peruviana Dillon \& Sagástegui * & $1-2$ & $2-3$ & $3-5$ & $9-11$ \\
\hline Achyrocline ribasiana Deble \& Marchiori & 3 & $2-3$ & $5-6$ & $14-15$ \\
\hline Achyrocline rupestris Cabrera* & $4-5$ & $1-2$ & $5-7$ & $9-10$ \\
\hline Achyrocline satureioides (Lam.) DC. & $3-6$ & $1-3$ & $4-9$ & $10-14$ \\
\hline Achyrocline scandens V. M. Badillo* & 4 & 2 & 6 & $13-14$ \\
\hline Achyrocline tomentosa Rusby & $4-5$ & $1-2$ & $5-7$ & $8-9$ \\
\hline Achyrocline venosa Rusby * & $3-4$ & $0-1$ & $4-5$ & $9-10$ \\
\hline
\end{tabular}

* espécies extra-brasileiras.

** com base na diagnose original.

Dillon M. O., Sagástegui, A. A new species of Achyrocline (Inuleae-Asteraceae) from Peru. Phytologia, New York, v. 60, n. 2, p. 106-110, 1986.

Dillon M. O., Sagástegui, A. Sinopsis de los géneros de Gnaphaliinae (Asteraceae-Inuleae) de Sudamérica. Arnaldoa, Trujillo, v. 1, n. 2, p. 591, 1991.

Freire, S. E. Asteraceae. Tribu IV. Inuleae. Flora Fanerogámica Argentina, Córdoba, n. 14, p. 160, 1995.

Giangualani, R. N. Las especies argentinas del género
Achyrocline (Compositae). Darwiniana, San Isidro, v. 20, n. 3-4, p. 549-576, 1976.

Hilliard, O. M., Burtt, B. L., Some generic concepts in Compositae-Gnaphaliinae. Bot. J. Linn. Soc., n. 82, p. 181-232, 1981.

Hind, D. J. N. Notes on the Compositae of Bahia, Brazil: I. Kew Bull., Kew, v. 48, n. 2, p. 254257, 1993.

Leins, P. Pollensystematische studien an Inuleen 1. Tarchonanthinae, Plucheinae, Inulinae, Buphthalminae. Bot. Jahrb. Syst., Leipzig, v. 91, n. 2, p. 91-146, 1971. 
Leins, P. Pollensystematische studien an Inuleen 2. Filagininae. Bot. Jahrb. Syst., Leipzig, v. 93, n. 4, p. 603-611, 1973.

Mattfeld, J. Notizbl. Botan. Gart. Mus., Berlin, Dahlem, n. 9, p. 381-385, 1925.

Nesom, G. L. Two new species of Achyrocline (Asteraceae: Inuleae) from Mexico. Phytologia, New York, v. 68, n. 5, p. 363-365, 1990.

Robinson, H. New species of Chionolaena and Stenocline from Brazil (Inuleae: Asteraceae). Phytologia, New York, v. 55, n. 3, p. 121-125, 1984.

Zardini. E. M. Revisión del genero Noticastrum (Compositae-Astereae). Rev. Mus. La Plata (Nueva Serie), La Plata, v. 13, n. 83, p. 313-424, 1985.

\section{INDICE DE NOMES CIENTÍFICOS}

Achyrocline, 10,11, 12, 14, 15, 16 alata, $11,12,15,16,17$ anabelae, $11,12,13,15,16,17$ arrojadoana, $11,13,15,17$ brittoniana, $11,15,17$ celosioides, 11 chionaea, 10,16 crassiuscula, 12, 16, 17

disjuncta, 11, 13, 15, 16, 17

eriodes, 10,16

flaccida, 11, 12, 15, 17

gardneri, 10,16

gaudens, 17

gertiana, 11, 12, 13, 14, 15, 17

glandulosa, 11, 12, 13, 15, 17

guerreroana, 14, 15, 17

heringeri, 10,16

hyperchlora, 11, 12, 15, 17

latifolia, 17

luisiana, $11,12,13,14,16,17$

madioides, 11, 16

marchiorii, 11, 12, 15, 16, 17

oaxacana, 15,17

peruviana, 17

ribasiana, $11,12,13,16,17$

rufescens, 12

rugosa, 16

rupestris, 11,17

satureioides, $11,12,13,15,16,17$

satureioides, 16

scandens, 11,17

tomentosa, 11, 12, 13, 15, 16, 17

venosa, $11,15,17$

Edmondia, 15

Helichrysopis, 15

Helichrysum, 15

Pseudognaphalium, 10, 11, 15

Stenocline, 10,14 chionaea, 10,16

Chionolepis, 10,11, 14 eriodes, 10,16

Eustenocline, 10

gardneri, 10,11, 16

heringeri, 10, 16

Tricephalum, 10

Stenophalium, 10,11, 12, 14, 15, 16

almasense, 11,16

chionaeum, $11,12,13,14,15,16,17$

eriodes, $11,12,13,14,15,16,17$

gardneri, $11,12,13,14,15,16,17$

heringeri, $11,12,14,16,17$

Syncarpha, 15

APÊNDICE

Material examinado:

1. Achyrocline alata (H. B. K.) DC.

BRASIL: RIO GRANDE DO SUL: Caxias do Sul, Santa Lúcia do Piaí, em capoeira, A. Kegler 957, 1.IV.2000 (MBM 267.892). Jaquirana, p. Bom Jesus, na beira da estrada, perto de córrego, L. P. Deble, A. S. de Oliveira \& Marchiori, 7.IV.2004 (HDCF).

2. Achyrocline anabelae Deble

BRASIL: RIO GRANDE DO SUL: Bagé, Rincão do Inferno, em afloramento rochoso, na orla da mata, L. P. Deble \& A. S. de Oliveira, 4.XI.2004 (MBM, tipo de $A$. anabelae); Casa de Pedra, sobre rocha, em solo arenoso raso, topo de rochedo, em sol pleno, flores amarelas, I. Fernandes 660, 8.X.1988 (ICN).

3. Achyrocline arrojadoana Mattf.

BRASIL: RIO DE JANEIRO: Itatiaia, planalto, 2.400 m.s.m., H. Sick \& L. F. Pabst, 17.IV.1965 (PEL).

4. Achyrocline brittoniana Deble \& Marchiori BOLIVIA: LA PAZ: La Paz, entre El Alto y Pocota, 4.100 m.s.m., A Cevallos 52 et al., II. 1979 (SI). Murillo, hills along west side of alto seguencoma, 3.400 m.s.m., $16^{\circ} 32^{\prime}$ 'S $68^{\circ} 05^{\prime}$ W, J. C. Solomon, 18.IV.1987 (MO, MBM).

5. Achyrocline chionaea (DC.) Deble \& Marchiori BRASIL: RIO DE JANEIRO: Nova Friburgo, nas rochas, 1.200 m.s.m., R. Franzen 45, 13.VI.1999 (MBM 239.428). MINAS GERAIS: Caparaó, Parque Nacional do Caparaó, p. Cachoeira Bonita, 1700-1800 m.s.m., G. Hatschbach 55.476 et al., 14.VI.1991 (MBM 143.756). Conceição do Mato Dentro, 3-5 km ao sul de, campo rupestre, G. Hatschbach 52.873 \& V. Nicolack, 18.V.1989 (MBM 128.660).

6. Achyrocline crassiuscula (Malme) Deble \& Marchiori BRASIL: RIO GRANDE DO SUL: Rio Grande, em dunas de areia, próximo ao Taim, J. N. C. Marchiori, 14.IV.2004 (HDCF).

7. Achyrocline disjuncta Hemsl.

BRASIL: ESPÍRITO SANTO: Ilha de Trindade, no alto do pico Trindade, encosta norte, 600 m.s.m., subarbusto de $120 \mathrm{~cm}$, de copa frondosa e capítulos brancos, R. J. V. Alves 4.447, 07.VIII.1994 (MBM 282.853).

8. Achyrocline eriodes (Mattf.) Deble \& Marchiori BRASIL: BAHIA: Rio de Contas, Pico das Almas, vertente leste, trilha da faz. Silvina-Queiroz, $13^{\circ} 32^{\prime}$ S, $41^{\circ} 56 \mathrm{w}$, 
1350-1450 m.s.m., campo arenoso com afloramento de rocha conglomerada, brácteas involucrais amarelo-claro, R. M. Harley 25.789 et al., 30.X.1988 (MBM).

9. Achyrocline flaccida (Weinm.) DC.

BRASIL: RIO GRANDE DO SUL: Bagé, BR 153, cerca de $10 \mathrm{~km}$ da entrada da cidade, em campo, L. P. Deble \& A. S. de Oliveira, 13.III.2004 (HDCF). Caxias do Sul, Vila Seca, capoeira, A. Kegler 717, 19.II.2000 (HUCS 15.210). SÃO PAULO: São Paulo, alto da Lapa, W. Hoehne, 13.III.1947 (SPF 14.063).

10. Achyrocline gardneri (Baker) Deble \& Marchiori BRASIL: MINAS GERAIS: Constantino da Mata, dos afloramentos rochosos, capítulos alvacentos, G. Hatschbach 30.211, 12.VIII.1972 (MBM 23.215). Diamantina, $20 \mathrm{~km}$ ao sul de, serra na estrada para Conselheiro da Mata, P. E. Gibbs, R. Abbott \& J. B. de Andrade, 17.V.1977 (MBM 62.100).

11. Achyrocline gertiana Deble \& Marchiori

BRASIL: MATO GROSSO DO SUL: Nhecolândia, fazenda Nhumirim, erva do ecótono, com $2 \mathrm{~m}$, flores amareladas, freqüente, F. Chagas \& Silva 767, 31.VII.1985 (MBM, tipo de A. gertiana).

12. Achyrocline glandulosa Blake

ARGENTINA: CHACO: $1^{\circ}$. DE MAJO, Margarita Belén, colônia Benitez, espinillar, A. G. Schulz 19.052, 11. IV. 1978 (SI). SALTA: Salta, Quebrada de San Lorenzo, 1450-1800 m.s.m., L. J. Novara \& S. B. Bruno 8.980, 19.VI.1989 (SI). BRASIL: SANTA CATARINA: Palhoça, campo do Maciambu, em restinga, R. Reitz \& Klein 345, 12.III.1953 (HBR 14.648). RIO GRANDE DO SUL: Torres, Itapeva, erva em borda de banhado, capítulos amarelos, J. A. Jarenkow 2088, 24.III.1992 (PEL 13.836).

13. Achyrocline heringeri (H. Rob.) Deble \& Marchiori BRASIL: DISTRITO FEDERAL: Brasília, Bacia do Rio São Bartolomeu, erva com pubescência esbranquiçada, capítulos amarelos, E. P. Heringer 7.055, T. S. Filgueras, R. C. Mendonça \& A. S. Pereira (fotótipo IBGE! US!).

14. Achyrocline hyperchlora Blake

ARGENTINA: JUJUY: Jujuy, Sierra de Zapla, cerro de la Cruz, A L. Cabrera 32.618, S. Botta, N. Deginani \& F. O. Zuloaga, 11.V.1981 (SI).

15. Achyrocline latifolia Weddel

BOLIVIA: LA PAZ: Murillo, por el camino a Achocalla, zona arbustiva, semi-arida, 3.900 m.s.m., $16^{\circ} 34^{\prime} \mathrm{S}$ $68^{\circ} 11^{\prime}$ W, J. C. Solomon 16.566, 18.IV.1987 (MO, MBM). 16. Achyrocline luisiana Deble

BRASIL: RIO GRANDE DO SUL: Sapucaia do Sul, morro Sapucaia, flores amarelas, comum no topo do morro, 290 m.s.m., I. Fernandes 637, 26.XI.1989 (ICN, tipo de A. luisiana).

17. Achyrocline madioides Meyen \& Walp.

BOLIVIA: COCHABAMBA: Cochabamba, bosque de Polylepis, 3800 m.s.m., cabecera de Valle, bosque montano, I. Hensen 461, 25.X.1989 (SI).

\section{Achyrocline marchiorii Deble}

BRASIL: RIO GRANDE DO SUL: Alegrete, Cerro do Tigre, na subida para o topo, abundante, crescendo entre rochas, brácteas amarelo-limão, L. P. Deble, A. S. de Oliveira \& J. N. C. Marchiori, 28.X.2004 (MBM, tipo de $A$. marchiorii).

19. Achyrocline ribasiana Deble \& Marchiori

BRASIL: ESPÍRITO SANTO: Cachoeira do Itapemirim, Vargem Alta, morro do Sal, arenícola, heliófila, flores alvas, folhas discolores, V. F. Ferreira 1847, 16.VIII.1981 (MBM, tipo de A. ribasiana).

\section{Achyrocline rupestris Cabrera}

ARGENTINA: JUJUY: Rinconada, mina pirquitas, camino a Cusi-Cusi, H. Schwabe 1.136 et al. (LP). SALTA: Los Andes, San Antonio de los Cobres, Quebrada de Polvorilla, 4.000 m.s.m., A L. Cabrera 8.395, 2.III.19444 (LP, tipo de $A$. rupestris).

21. Achyrocline satureioides (Lam.) DC.

BRASIL: RIO GRANDE DO SUL: Alegrete, Cerro do Tigre, na estrada, em solo arenoso, L. P. Deble, A. S. de Oliveira \& Marchiori, 1.IV.2004. Bagé, p. Aceguá, BR 153, na beira da estrada, solo pedregoso, L. P. Deble \& A. S. de Oliveira, III.2004 (HDCF). São José dos Ausentes, cânion do Monte Negro, cerca de 1.300 m.s.m., L. P. Deble, A. S. de Oliveira \& Marchiori, 7.IV.2004 (HDCF).

22. Achyrocline tomentosa Rusby

BRASIL: RIO GRANDE DO SUL: São José dos Ausentes, na orla da matinha nebular, p. Monte Negro, cerca de 1.300 m.s.m., L. P. Deble, A. S. de Oliveira \& Marchiori, 7.IV.2004 (HDCF).

23. Achyrocline venosa Rusby

ARGENTINA: JUJUY: Valle Grande, serrania de Calilegua, colorado, H. A Fabris 5786 et al. (LP). 